J3eA, Journal sur l'enseignement des sciences et technologies de l'information et des systèmes,

Volume 3, Hors-Série 1, 4 (2004)

DOI : http://dx.doi.org/10.1051/bib-j3ea:2004604

(C) EDP Sciences, 2004

Perte de vision centrale : aide optique et électronique portée sur la tête

J.C. Kastelik, M. Pommeray et M. Slachciak

Institut d'Électronique, de Microélectronique et de Nanotechnologies

UMR CNRS 8520

Département Opto-Acousto-Électronique

Université de Valenciennes

Le Mont Houy

F-59313 Valenciennes CEDEX 9, France

Jean-Claude.Kastelik@univ-valenciennes.fr 


\title{
Perte de vision centrale : aide optique et électronique portée sur la tête
}

\author{
Jean-Claude Kastelik, Michel Pommeray, Marc Slachciak \\ Institut d'Electronique, de Microélectronique et de Nanotechnologies, U.M.R. \\ C.N.R.S. 8520, Département Opto-Acousto-Electronique \\ Université de Valenciennes, Le Mont Houy, 59313 Valenciennes cedex 9 \\ Jean-Claude.Kastelik@univ-valenciennes.fr
}

\begin{abstract}
Résumé - Cet article décrit les premiers essais d'un système d'aide visuelle optique et électronique porté sur la tête et destiné à des personnes présentant une perte sévère de la vision centrale, due principalement à la dégénérescence maculaire liée à l'âge. Les principales caractéristiques de cette pathologie, ses conséquences fonctionnelles et les situations de handicap résultantes sont présentées. Le système d'aide visuelle ainsi que les premiers essais par des personnes atteintes sont détaillés et discutés.
\end{abstract}

Mots clés : Handicap visuel, dégénérescence maculaire, aide visuelle portée sur la tête.

\section{INTRODUCTION}

La perte de vision centrale se traduit par des difficultés importantes dans l'accomplissement des tâches les plus élémentaires de la vie quotidienne. Ce type de pathologie est largement répandu dans les pays industrialisés du fait de l'allongement de la durée de vie. Ainsi la dégénérescence maculaire liée à l'âge (DMLA) est actuellement la principale cause de handicap visuel $[1,2]$. La prévalence [3] est estimée à 1,5\% dans la tranche d'âge 55 à 65 ans, $12 \%$ entre 65 et 75 ans et $30 \%$ entre 75 et 85 ans, ces chiffres étant hélas amenés à augmenter, en raison du vieillissement de la population. Aucun traitement ne permet de guérir de cette maladie. La D.M.L.A. pose donc un problème de santé publique majeur.

Les paragraphes qui suivent donnent tout d'abord les principales caractéristiques cette atteinte du système visuel et ses conséquences fonctionnelles. Un système d'aide visuelle porté sur la tête est ensuite détaillé, suivi des essais par des personnes atteintes. Ce projet pluridisciplinaire a été initié dans le cadre de l'Institut Régional (Nord-Pas de Calais) de Recherche sur le Handicap et implique plusieurs équipes des services d'Ophtalmologie et de Gériatrie du C.H.R.U. de Lille.

\section{CONSEQUENCES FONCTIONNELLES DE LA D.M.L.A., SITUATIONS DE HANDICAP}

La partie centrale de la rétine ou « macula », est caractérisée par une forte densité de cellules photosensibles assurant ainsi une grande précision de la vision. Bien que très petite puisque représentant de l'ordre de 2 à $3 \%$ de la surface totale de la rétine, la quasi-totalité des informations visuelles traitées par le cerveau provient de la macula. La dégénérescence maculaire altère cette partie centrale sous des formes différentes, dont la plus répandue ( $80 \%$ des cas) est la forme atrophique (dite « sèche »), d'évolution lente et progressive. La vision périphérique reste normale. Des traitements [4] sont apparus ces dernières années pour la forme exsudative principalement, visant essentiellement à limiter l'extension de la maladie.

Les conséquences sur la perception visuelle dépendent du degré d'avancement de la D.M.L.A.. Pour les formes débutantes, la présence de petits scotomes paracentraux se traduit par une disparition de certaines lettres des mots au voisinage du point de fixation et rend la lecture pénible, lente et fatigante. L'extension de ces scotomes perturbe l'accomplissement des différentes tâches de la vie quotidienne : reconnaissance des visages, des formes et des inscriptions à moyenne distance, conduite automobile, observation d'un écran de télévision...La figure 1 tente de simuler ce défaut de perception. Par contre, la vision péricentrale et périphérique est conservée, ce qui permet la plupart des activités déambulatoires. Dans les formes intermédiaires, l'évolution de la lésion entraîne la formation d'un scotome central d'étendue dépassant deux degrés d'angle visuel. Le patient se définit un point de fixation excentré ou sur un îlot résiduel de rétine saine situé dans une zone atrophique. La gêne est plus importante : la vision d'ensemble est conservée mais la partie centrale est floue. Dans les formes évoluées, la taille du scotome peut atteindre jusqu'à trente degrés de diamètre. L'acuité visuelle de loin est inférieure à 0,05 , ce qui correspond, en France, à la cécité légale. Même la vision périphérique devient peu utilisable.

Plusieurs études concernant l'impact psychosocial [5], la perception des tests visuels [6] et l'identification des activités journalières $[7,8]$ posant problème ont mis en évidence la forte dégradation des conditions de vie due à la perte d'autonomie des personnes atteintes de D.M.L.A.. Six principaux domaines d'activités de priorité équivalente peuvent être distingués :

- Achats : Localiser un rayon, choisir un produit, lire le prix , payer...

- Cuisine, Ménage : Lire une recette, éplucher, peser, utiliser des appareils électroménagers, prendre ses repas, faire la vaisselle...

- Déplacement, transport : Lire le nom des rues, éviter les obstacles peu contrastés, acheter un ticket, prendre l'escalier, l'escalator...

- Hygiène, santé : faire sa toilette, se raser, prendre ses médicaments...

- Vie sociale : Lire le journal, écrire, gérer ses comptes, remplir des formulaires, reconnaître des visages, regarder la télévision... 
- Bricolage, jardinage : Coudre, clouer, planter, semer...

\section{AIDE VISUELLE OPTIQUE ET ELECTRONIQUE PORTEE SUR LA TETE.}

L'idée d'un système d'aide visuelle porté sur la tête est intéressante $\mathrm{du}$ fait de l'évolution rapide des technologies et des techniques numériques qui ouvre de nouvelles possibilités d'acquisition, de traitement et de restitution d'images. Un tel système se compose d'une caméra à capteurs à transfert de charges munie d'un objectif approprié se substituant à la vision naturelle. Elle intègre les fonctions de mise au point automatique sur la scène visée par la personne et de stabilisation d'images indispensable pour compenser les mouvements de la tête.

Les traitements d'images sont effectués en fonction de la scène observée et du degré d'atteinte de l'oeil du patient. Le traitement le plus intuitif consiste à effectuer des effets de zoom sur l'image captée. Cet effet peut être obtenu de plusieurs manières : soit à l'aide d'un zoom optique variable (jusqu'à une valeur supérieure à 10) monté directement sur la tête de caméra, soit à l'aide d'un traitement électronique (de l'ordre de 2 à 4), soit par combinaison des deux. Les traitements électroniques des images sont effectués de manière déportée dans un premier temps, c'est à dire que les images fournies par la caméra sont transférées vers un ordinateur qui effectue les calculs et retourne les images traitées vers le dispositif de restitution. La technique la plus utilisée pour l'affichage est celle de l'imagerie virtuelle : l'œil observe l'image d'un microécran au travers d'une optique grossissante. La technologie la plus mature industriellement pour ce type d'afficheur est celle des matrices actives à cristaux liquides.

L'identification du domaine de vision optimal des patients atteints de D.M.L.A. est indispensable pour appliquer aux images recueillies par la caméra les transformations nécessaires pour une perception améliorée (analyse du champ visuel, étude de la sensibilité aux contrastes, aux couleurs, fréquences spatiales et temporelles). Les personnes atteintes de D.M.L.A. développent également des stratégies d'exploration visuelle visant à compenser leur handicap [9]. Se pose également le problème de la compatibilité entre les fonctions de localisation assurées, chez un sujet sans déficience visuelle, par la rétine périphérique et celles de reconnaissance assurées par la partie centrale. De nombreuses tâches nécessitent ces deux types d'information. L'idée naturelle d'agrandir l'image réduit le champ périphérique, ce qui peut entrainer des perturbations des fonctions de localisation (vertiges, perte d'orientation). Des solutions peuvent être envisagées dans la conception du système d'aide porté sur la tête : soit un afficheur monoculaire, l'autre œil restant en vision naturelle, soit préserver une zone de vision périphérique autour d'une partie centrale agrandie ; d'où la réalisation d'une maquette d'aide visuelle donnant la possibilité d'essayer ces différentes configurations.
Finalement, le système utilisé, représenté à la figure 2, pour les essais est composé d'un casque de visualisation (type Head Mounted Display) à afficheurs à cristaux liquides. Il a été modifié de façon à présenter au patient une restitution monoculaire ou binoculaire. Un bloc caméra CCD intégrant les fonctions de zoom motorisé, de mise au point automatique, de stabilisation d'images est fixé au dessus du casque. Un ordinateur déporté pour effectuer le pilotage et les réglages de la caméra nécessaires lors des tests. Plusieurs modes de fonctionnement sont possibles : restitution des images acquises par la caméra en direct ou en différé si des traitements électroniques sont appliqués, restitution directe des séquences d'images correspondant aux situations types avec ou sans traitement électronique.

\section{ESSAIS CLINIQUES}

Ces premiers essais ont été réalisés dans l'appartement thérapeutique de l'Hôpital « Les Bateliers ", pièce aménagée comportant en particulier une cuisine intégrée permettant ainsi de travailler dans les conditions de la vie quotidienne. Les objectifs visés étaient essentiellement de recueillir les premières impressions de personnes atteintes de D.M.L.A. portant un tel système dans plusieurs configurations. Trois dames âgées respectivement de 67, 73 et 80 ans ont accepté de participer. Toutes trois présentaient une D.M.L.A. dans un stade avancé puisque l'acuité visuelle était inférieure à $1 / 10$ pour le meilleur œil et à $1 / 20$ pour l'autre. Deux patientes présentaient une D.M.L.A. récente (1998) : une forme exsudative stabilisée par traitement laser et une forme atrophique. La troisième patiente présentait une DMLA depuis 1992 dans sa forme exsudative également stabilisée par traitement laser. Toutes les trois étaient en bonne forme physique, très motivées et disponibles pour ces essais. Chacune d'elles a ainsi participé à une séance d'environ 2 heures.

Tout d'abord, une discussion a permis de présenter le système d'aide visuelle et les essais envisagés, de mieux connaître les besoins particuliers de ces patientes ainsi que les systèmes de suppléance déjà utilisés, le cas échéant. Les réponses fournies concernant les difficultés rencontrées dans la vie quotidienne ont confirmé la classification présentée auparavant : la demande essentielle se situe en une aide à la lecture, à l'écriture et à la reconnaissance des visages. Ces patientes essaient également de compenser leur déficience visuelle : ainsi l'écriture d'un mot ou d'une phrase se fait d'un seul jet sans relever la pointe du crayon rendant impossible le remplissage d'un chèque par exemple, l'observation d'un écran de télévision se fait très proche de l'écran, ce qui occasionne de la fatigue rapidement, l'épluchage d'un légume est possible mais imparfait car n'utilisant que le toucher, la diminution de la sensibilité au contraste se traduit pour l'une d'elles de la manière suivante : elle préfère sortir en fin d'après-midi car la vision des feux tricolores est meilleure. Comme moyens de suppléance, deux personnes utilisent un téléagrandisseur efficace pour la lecture, la troisième un système télescopique de Képler difficile à utiliser du fait du fort grossissement d'où un champ visuel réduit et une 
amplification des mouvements rendant difficile la visée et la lecture globale d'un mot.

\section{A. Lecture d'une échelle d'acuité visuelle}

Cette échelle permet l'évaluation en vision de près (1 $\mathrm{m})$ et de loin $(4 \mathrm{~m})$ Le premier problème à résoudre pour les patientes est celui de la visée. En effet l'axe de la caméra est déportée par rapport à l'axe du regard et donc une courte phase d'adaptation s'est avérée nécessaire. L'acuité visuelle a été mesurée tout d'abord pour chaque œil, l'autre étant caché, sans puis avec l'appareil en modifiant le grandissement. Cette expérience a été renouvelée pour une lecture avec les deux yeux. Les valeurs d'acuité mesurées sont conformes à celles établies dans le cadre d'une consultation d'ophtalmologie basse vision classique vis à vis de l'amélioration en fonction du grossissement appliqué. Nous avons cependant noté les points suivants : il n'y pas de différence de mesure d'acuité entre celles obtenues pour le meilleur œil en vision monoculaire par rapport à celles obtenues en immersion totale. Ceci s'explique par le fait que la restitution en immersion totale n'est pas binoculaire puisque la même image est affichée devant les deux yeux. De plus la dissymétrie d'atteinte visuelle est forte entre l'œil gauche et l'œil droit, d'où prédominance de l'œil le moins atteint. On peut noter également une légère amélioration de l'acuité avec le casque sans effet de zoom par rapport à l'acuité mesurée sans l'appareil, ce qui est du à un meilleur contraste apporté par les afficheurs.

\section{B. Test de lecture}

Il s'agit d'un test classique de lecture Keeler (Série A) conçu pour permettre une estimation rapide $\mathrm{du}$ grossissement nécessaire à la lecture de journaux, le patient étant corrigé pour $25 \mathrm{~cm}$. La lecture sans appareil indique un grossissement de l'ordre de 10. Avec le casque, nous avons mis en évidence quelques difficultés liées à l'encombrement du dispositif, en particulier le déport de la caméra vers l'avant qui rend difficile une posture normale pour une situation de lecture. De plus, pour des valeurs élevées de grossissement (supérieures à 4 ) en vision de près (distance inférieure à $30 \mathrm{~cm}$ ), la mise au point automatique de la caméra est difficile. Un avantage du système par rapport à une aide optique classique est le plus grand champ visuel même à fort grossissement, ce qui permet à la personne de lire un mot globalement plutôt que de scruter chacune des lettres les unes après les autres. De plus, en effectuant un arrêt sur l'image du mot, la vision est beaucoup plus confortable

\section{Déambulation}

Il s'agit d'effectuer un petit déplacement selon un parcours de quelques mètres en direction d'affichettes simulant un nom de rue. En immersion totale sans grandissement, le déplacement s'effectue avec difficulté du fait que le champ de la caméra ne recouvre pas les pieds de la personne et la lecture des affichettes n'est possible que de près (environ $40 \mathrm{~cm}$ ). La déambulation en immersion totale avec grandissement ( 3 dans ce cas) est à proscrire : risque important de chute, de vertige. Lorsque le système est configuré en monoculaire avec grandissement sur le meilleur oeil, la personne ressent au départ une certaine gêne du fait d'un conflit entre les deux images perçues : l'une en vision naturelle et l'autre agrandie par l'afficheur. La démarche est cependant plus franche : la personne utilise l'œil non appareillé pour se repérer et l'autre pour lire les affichettes ( à une distance d'environ $1,5 \mathrm{~m}$ pour un grandissement de 3).

\section{Test d'écriture}

Pour ce test, il est demandé à la patiente d'écrire quelques mots, de remplir un chèque ou de placer sur un dessin, figurant une échelle, les barreaux entre les deux montants. Dans ces configurations où la personne est assise, le port $\mathrm{du}$ casque en immersion totale est le plus confortable. Le grossissement de l'image à l'aide du zoom améliore de manière significative la qualité de l'écriture (meilleur repérage des cases dans le cas du remplissage d'un chèque, écriture des mots plus soignée). Le problème majeur pour l'écriture est la maîtrise du geste du à une perception des mouvements amplifiés par le grossissement. Comme le montre le test des barreaux d'échelle représenté à la figure 3. L'écriture avec un zoom améliore la précision du tracé, en particulier pour un zoom égal à 2 ou l'on observe que les barreaux, dessinés de gauche à droite sont bien attachés au montant alors que de l'autre côté, ils le dépassent largement. La personne arrive à maîtriser le point de départ mais comme l'amplitude du tracé qu'elle effectue est perçue de manière amplifiée, le contrôle du point d'arrivée est plus difficile. Ce défaut pourrait être corrigé, pour des grossissements faibles, après une période d'apprentissage.

\section{CONCLUSION}

Le travail présenté dans cet article met en évidence les difficultés à surmonter pour l'élaboration d'un système d'aide porté sur la tête par des personnes présentant une atteinte de la vision centrale. Ces difficultés sont liées aux multiples compétences nécessaires pour mener à bien ce projet : tout d'abord sur le plan des techniques et technologies en électronique, optique et informatique ; ensuite dans les disciplines propres au monde médical : ophtalmologie et basse vision, optique physiologique, gériatrie, rééducation... Cette première étude a ainsi permis d'établir des collaborations entre équipes complémentaires.

Sur le plan des technologies, les perspectives semblent tout à fait favorables à la réalisation d'un système plus léger, compact et ergonomique. Les dispositifs d'acquisition et de restitution d'images ne cessent de progresser avec, en particulier, l'émergence de nouvelles technologies prometteuses comme les microécrans émissifs pour lesquels des performances très intéressantes sont annoncées : flux lumineux plus important, émission 
lambertienne, encombrement et poids réduits, consommation moindre.

Du point de vue de l'utilisateur, les premiers essais se sont révélés très encourageants malgré l'emploi d'une maquette d'évaluation lourde et encombrante. Ces essais ont ainsi reçu un accueil très favorable de la part des personnes atteintes : en premier lieu, il existe une demande forte pour un système d'aide visuelle parce que le handicap résultant de la D.M.L.A. est sévère. La perte d'autonomie dans les tâches les plus élémentaires de la vie quotidienne est importante et il est urgent d'y remédier. A partir des tests effectués, des évolutions peuvent d'ores et déjà être envisagées. Ainsi la configuration dite en " immersion totale » pour la déambulation est à proscrire à cause des risques de chute accentués par l'âge des patients. On peut ainsi envisager, plutôt qu'un bloc caméra intégrant un zoom optique motorisé, une simple lentille avec un grossissement fixé complété par un zoom réalisé électroniquement; d'où un gain significatif en poids, encombrement et consommation électrique. Pour la restitution, un dispositif sachant «se faire oublier » lors de certaines tâches, en particulier la déambulation, serait plus indiqué. L'appareil pourrait comporter un afficheur amovible mécaniquement ou électro-optiquement (transparence de l'écran « seethrough »).

Concernant les traitements à appliquer sur les images fournies par la caméra, seuls les effets de grossissement ont pu être testés. Mais d'autres traitements sont à discuter en fonction des capacités visuelles résiduelles du patient. C'est sur ce point que les recherches doivent être approfondies avec l'identification du domaine de perception visuelle optimale, l'étude de la compensation de la perte de vision centrale par la vision périphérique, la sensibilité aux couleurs, aux contrastes, les problèmes de vertige liés à la perception d'une image agrandie. La souplesse qu'apporte la possibilité de (re)programmer les composants sans changer la configuration matérielle est très utile pour une adaptation du système au cas par cas et en fonction de l'évolution de la pathologie.

La validation des améliorations apportées au système doit être effectuée le plus souvent possible par les utilisateurs. A plus long terme, cette démarche itérative permettra de converger vers un appareil d'aide visuelle répondant le mieux possible aux besoins des patients présentant une altération de la vision centrale Cependant, l'acceptation finale de ce système ne pourra être effective qu'au terme d'une phase d'apprentissage, de rééducation qui pourrait être mené avec la collaboration d'orthoptistes.

\section{REFERENCES}

[1] Klein R, Wang Q,. Klein BE,Moss SE et Meuer SM, " The relationship of age-related maculopathy, cataract, and glaucoma to visual acuity.», Invest. Ophtal. Vis. Sci., 1995, p.182-91.

[2] Williams RA, Brody BL, Thomas RG., Kaplan RM, Brown SI., « The psychosocial impact of macular degeneration », Arch. Ophtalmol., 116, 1998, p.514-20

[3] VanNewkirk MR, Nanjan MB, Wang JJ, Mitchell P et Mac Carthy CA, " The Prevalence of age-related maculopathy », J. of Ophtal.,107, 8, 2000, p 1593-600.

[4] Ciulla TA, Danis RP et Harris A, « Age-related macular degeneration : a review of experimental treatments » Survey of Ophtal.,43, 2, 1998, p 134-46

[5] Williams RA, Brody BL, Thomas RG, Kaplan RM, Brown SI, " The psychosocial impact of macular degeneration ”, Arch. Ophthalmol, 116 :514-20, 1998.

[6] Dahlin Ivanoff S, Sjöstrand J, Klepp KI, Axelsson Lind L, LundGren Lindqvist B, “ Planning a health education programme for the elderly impaired person - afocus group study ", Disability and rehabilitation, 18 :515-22, 1996.

[7] Kastelik JC, Pommeray , M, Pardessus V, Gaudefroy C, Dedès V, Charlier J. " Aide visuelle pour patients atteints de DMLA, Elaboration d'un catalogue de situations types " Handicap 2002 2ème conférence pour l'essor des technologies d'assistance p.105-108

[8] Weissenthaner L. "Analyse des besoins de la clientèle âgée atteinte de dégénérescence maculaire", 4ème Symposium scientifique sur l'incapacité visuelle et la réadaptation, Volume 4, Université de Montréal, pp 40-43, février 2001

[9] Mazoyer V, Knoblauch K, Fontanay, Koenig F et VitalDurand, « Identification of low-pass filtered images by low vision patients ", Visual Impairment Research, 1, 3, 1999, p. 187-92.

\section{REMERCIEMENTS}

Ce travail a bénéficié du soutien financier de la région Nord-Pas de Calais (02.0529, $\left.\mathrm{n}^{\circ} 02340056\right)$ et du FEDER (OBJ 1-2003/2003-4.1-27/1796). 

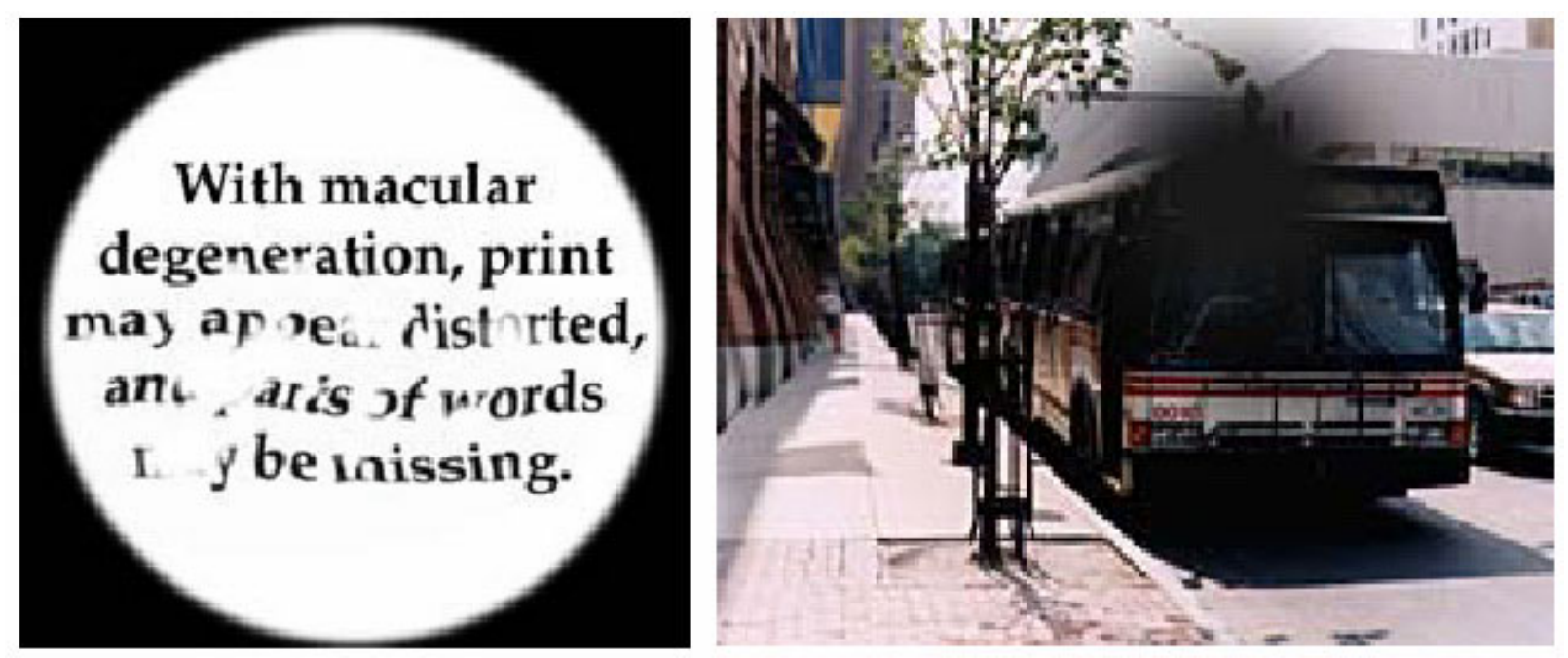

Fig.1. Simulation de la perception visuelle d'une personne atteinte de D.M.L.A. : Déformation, disparition de caractéres, tâche centrale.

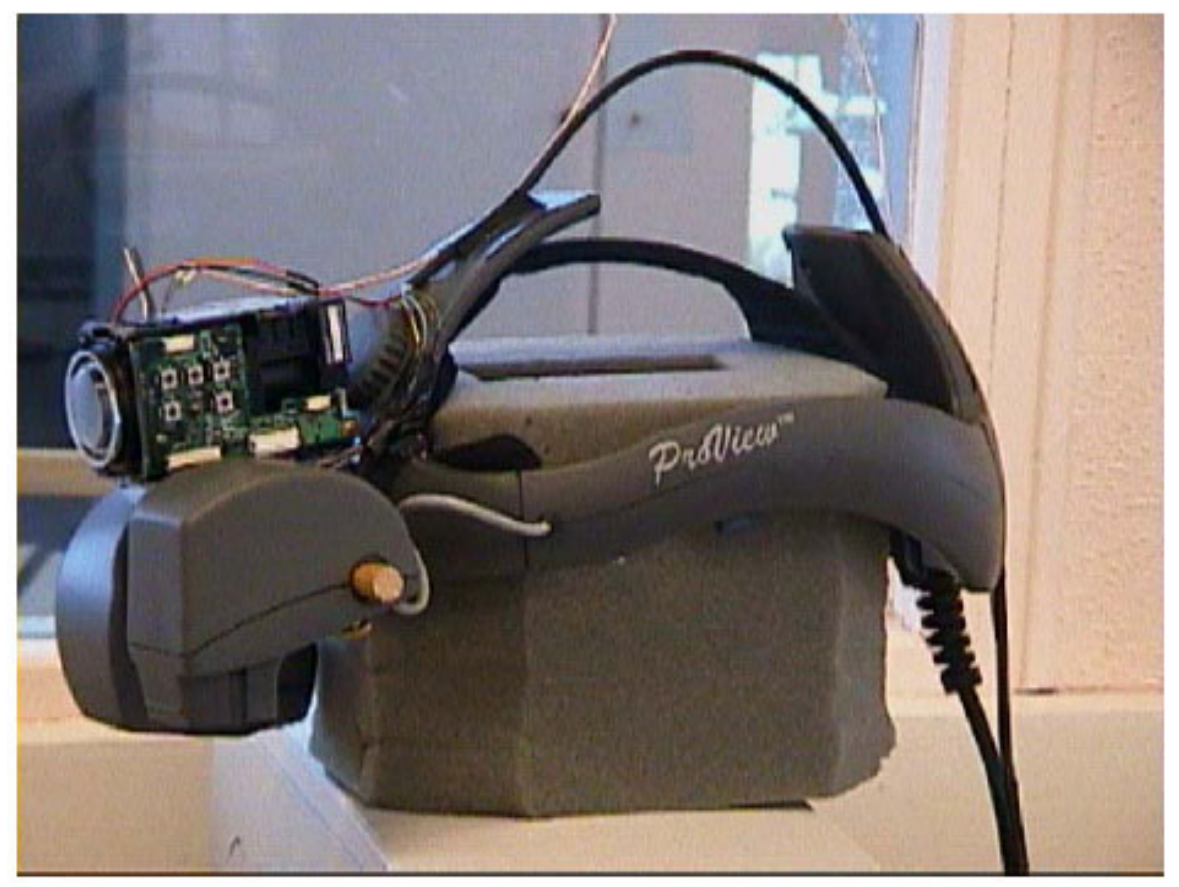

Fig.2. Photographie du système d'aide visuelle porté sur la tête. 


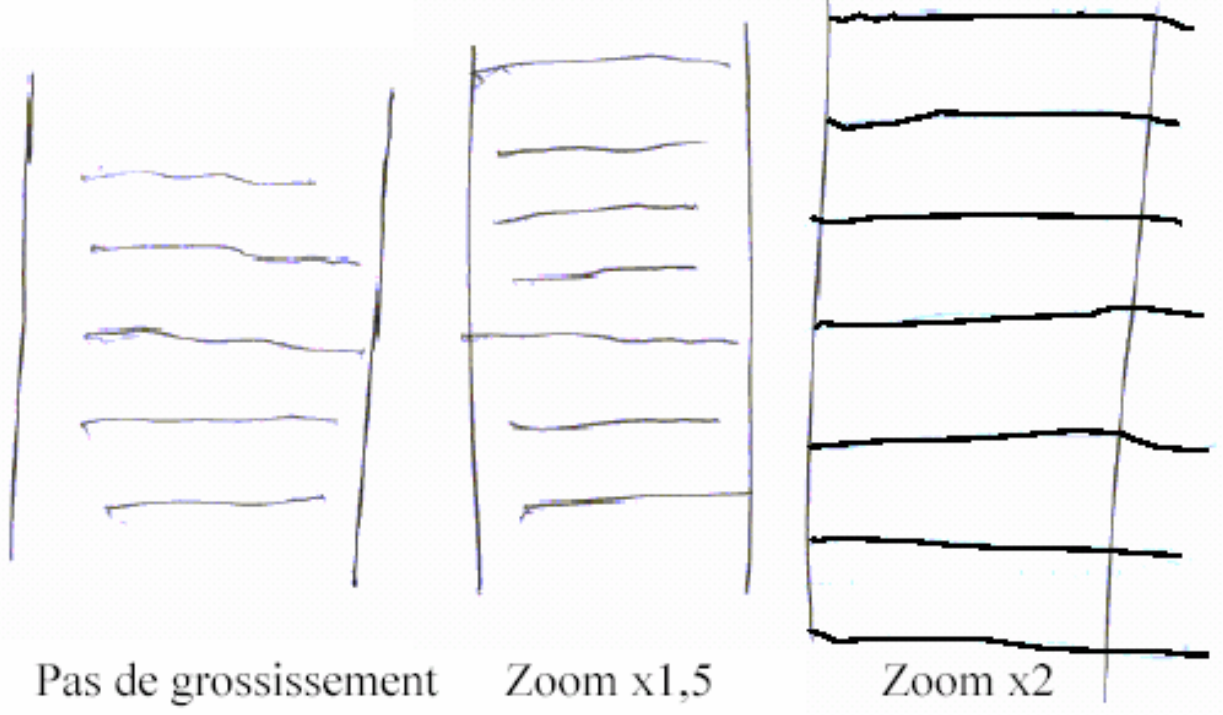

Fig.3. Test d'écriture des «barreaux d'échelle». 